Research Article

\title{
Unsupervised Wireless Network Model-Assisted Abnormal Warning Information in Government Management
}

\author{
Yumeng Sun (iD) \\ College of Environment, Education and Development, University of Manchester, Manchester, Great Manchester, UK \\ Correspondence should be addressed to Yumeng Sun; yumeng.sun-2@postgrad.manchester.ac.uk
}

Received 22 September 2021; Revised 12 October 2021; Accepted 13 October 2021; Published 26 October 2021

Academic Editor: Guolong Shi

Copyright ( 2021 Yumeng Sun. This is an open access article distributed under the Creative Commons Attribution License, which permits unrestricted use, distribution, and reproduction in any medium, provided the original work is properly cited.

\begin{abstract}
The data generated through telecommunication networks has grown exponentially in the last few years, and the resulting traffic data is unlikely to be processed and analyzed by manual style, especially detecting unintended traffic consumption from normal patterns remains an important issue. This area is critical because anomalies may lead to a reduction in network efficiency. The origin of these anomalies may be a technical problem in a cell or a fraudulent intrusion in the network. Usually, they need to be identified and fixed as soon as possible. Therefore, in order to identify these anomalies, data-driven systems using machine learning algorithms are developed with the aim from the raw data to identify and alert the occurrence of anomalies. Unsupervised learning methods can spontaneously describe the data structure and derive network patterns, which is effective for identifying unintended anomalous behavior and detecting new types of anomalies in a timely manner. In this paper, we use different unsupervised models to analyze traffic data in wireless networks, focusing on models that analyze traffic data combined with timeline information. The factor analysis method is used to derive the results of factor analysis, obtain the three major public factors and comprehensive factor scores, and combine the results with the BP neural network model to conduct a nonlinear simulation study on local governmental debt risk. A potential semantic analysis model based on Gaussian probability is presented and compared with other methods, and experimental results show that this model can provide a robust, over-the-top anomaly detection in a fully automated, data-driven solution.
\end{abstract}

\section{Introduction}

In wireless networks, the collected data includes different feature values and time axis. To find and detect anomalies, the values can be processed and modeled using an unsupervised algorithm [1]. By using unlabeled data, the algorithm assumes that the information about whether a factor is an outlier is unknown [2]. This is often the case, as anomalies in traffic data are rare and generally exist in multiple forms. The unsupervised algorithm automatically classifies and distinguishes between data structures and patterns [3]. Instead of directly detecting anomalous data, the algorithm describes and groups the data and then derives anomalous regions from these groups. The main advantage of this method is the ability to detect previously unforeseen or unanticipated anomalies. Another factor considered in wireless networks is the timeline [4]. When data is collected, this temporal information is usually collected along with it but is not widely used in the traditional anomaly detection process. However, network load fluctuates from day to day. For example, a normal value during a peak period may be an anomaly and not yet be detectable. Adding a timeline attribute to a model can detect periodic behavior [5].

Over the years, in order to speed up the economic and social development of the regions under their jurisdiction, governments around the world have been doing everything possible to increase their efforts to attract investment and raise the intensity of investment and construction, and many regions have even gone so far as to raise debt for construction and implement large-scale debt management, resulting in a large amount of hidden debt being incurred and accumulated [6]. In this process, the township government, which is at the bottom of the government system, bears a great responsibility for the risks arising from it and also bears a lot of economic development costs [7]. Particularly after the abolition of the agricultural tax, the township 
finance department is responsible for a large amount of public expenditure, but due to the backward economic situation of the rural areas, the income has not increased accordingly, and there is still a shortage of income and expenditure for construction, resulting in increasingly sharp contradictions between the income and expenditure of the township finance, the debt burden has gradually increased, and the financial risk has further accumulated [8]. According to the disclosure of relevant sources, in some areas of China, the financial risk of the township as a prelude to the occurrence of a payment crisis in the future of that government has developed to a very serious point, many economically backward townships, due to the uncertainty of a variety of economic factors and the impact of the financial system and means of the defects themselves, carrying a heavy financial burden; the township government can neither properly perform its role in organizing revenue and arranging [9]. They are also unable to guarantee the needs of regional construction and various public expenditure requirements, and regional economic development has been seriously affected. How to solve the problems and difficulties encountered in the development of township finance and maintain the stability and sustainable development of township government finance has become increasingly urgent, and the study of township finance risk has become a hot research topic in the relevant academic fields and an urgent task for the relevant functional departments [10]. Even many people still believe that if the township government has financial problems, they can only wait for the state to issue relevant policies to solve them, resulting in the financial risks at the lowest level not being given sufficient attention [11]. This thesis is based on the evaluation and prediction of township government finance in the current period from the perspective of the debt risk of township government and tries to provide useful ideas and ways to prevent and solve the financial risk of township government [12]. There are many different clustering algorithms, which differ in the criteria used to measure object similarity: distance, density, or statistical distribution; the most common and simplest clustering algorithm is $K$-means. Better detection methods combine statistical assumptions and clustering, such as the Gaussian mixture model (GMM), which assumes that all data points originate from some Gaussian mixture distribution.

Local governments are not yet aware of the necessity of establishing a local government debt risk early warning system, and there are still many problems with debt risk early warning, not only are there many shortcomings in the construction of the indicator system, but there is also a lack of risk early warning supervision, only individual local governments have established a risk early warning mechanism, and the debt risk information disclosure system is not sound, so it is inevitable that there is a lack of clear judgement on the size of local government debt risk. The establishment and implementation of a scientific local government debt risk early warning indicator system can not only provide a real-time understanding of the local government debt risk situation but also improve the openness and transparency of China's local government debt, thereby reducing local government debt risk and safeguarding the prosperity and stability of the financial system and the smooth operation of the economy. Based on the research of scholars at home and abroad, this paper constructs a local government debt risk early warning indicator system from four major aspects: local economic development indicators, local government revenue and expenditure indicators, local public risk indicators, and local government debt indicators. The factor analysis method is used to derive the results of factor analysis, obtain the three major public factors and comprehensive factor scores, and combine the results with the BP neural network model to conduct a nonlinear simulation study on local governmental debt risk.

\section{Related Work}

Most of the methods of machine learning can be classified as supervised or unsupervised. In anomalous text, supervised methods use a dataset that includes already labeled outliers. In order to separate the anomalies from the normal ones, the classification algorithms are trained based on the already labeled dataset. However, these algorithms are not applicable to unintended types of anomalies or to the derivation of new patterns. Because anomalies in telecommunications are rare and diverse, the resulting models do not detect subsequent anomalies. In addition, the cost of collecting training data is usually too high, as anomalies are usually manually flagged. In order to perform anomalous traffic detection, our research focuses on unsupervised methods [13]. The most effective methods are statistical- and clustering-based methods. In the statistical-based methods, a basic model assumption holds and outliers refer to points that are observed with a low probability. Most of these models are built based on Gaussian models. It is also possible to have anomalous parameter distributions, where normal points abnormally obey two different distributions [14]. In clustering methods, the aim is to separate data points and cluster data points with similar characteristics into groups. Each group is referred to as a cluster (clusters).

The similarity between objects can be defined in a parsing way. There are many different clustering algorithms, which differ in the criteria used to measure object similarity: distance, density, or statistical distribution; the most common and simplest clustering algorithm is $K$-means. Better detection methods combine statistical assumptions and clustering, such as the Gaussian mixture model (GMM), which assumes that all data points originate from some Gaussian mixture distribution. Estimates of the parameters are obtained by the EM algorithm, which aims to incrementally increase the log-likelihood value of the dataset through iterations. As mentioned in the references, several studies have used GMM for outlier detection problems [15]. Choosing the optimal number of clusters $K$ is not easy. Usually, different values are computed and then $K$ is manually selected and improved. Methods for automatic selection of the optimal $K$ values exist, and research results have been done to compare different algorithms. In telecommunication traffic data, the timeline is another factor to be considered. This information is referred to as contextual properties in 
some literature and can significantly improve the anomaly detection. Indeed, some values are considered normal in certain situations (peak hours) but anomalous in other situations (off-peak hours). One way to add a time axis is to introduce time series, such as cubic exponential smoothing and wavelet transforms [16]. A simpler approach is to create a specific model for each time period. An example would be to create a model for each hour of the day. Anomaly detection algorithms that combine the latter approach with Gaussian models have been studied. Another approach that takes the time axis into account is the initialized Gaussian mixture model-the GMM - which is a $K$ Gaussian mixture distribution, with each distribution accounting for different weights as time varies. The first application of this method to text mining is a hybrid classification distribution named Probabilistic Latent Semantic Analysis (PLSA) [17]. The technical term is called GPLSA (combined Gaussian distribution) and is commonly used in recommender systems. There are no articles on the use of GPLSA techniques for anomaly detection.

Artificial neural network early warning methods originated in the field of artificial intelligence, which is actually an operational model formed by a large number of interconnected neurons (or nodes). Each neuron can represent a specific class of output functions, which can also be called excitation functions [18]. Every arbitrary connection between two neurons represents a weight value of the signal passing through that connection. The output of the artificial neural network differs depending on the choice of connection, weight value, and type of output function of the network [19]. The early warning method does not require a strict distribution of data and has the ability to handle information omission. Comprehensive analysis of the above, using the integrated early warning method is more scientific and reasonable than the results derived from a single early warning method and can better ensure the effectiveness of the early warning results, and the government debt risk early warning model currently established in China mainly uses the linear relationship between indicators, while in fact, the early warning system is a multifaceted, nonlinear, and complex determination system [20]. Among the comprehensive early warning methods, the artificial neural network-based early warning method has the characteristics of nonlinearity, fault tolerance, and generalization ability compared with the other two early warning methods, which not only excludes the tendency of subjectivity but also can eliminate the factor of colinearity, and thus has more advantages [21].

\section{Unsupervised Wireless Network-Based}

3.1. Unusual Warning of Governmental Debt Risk. Being able to accurately predict the local governmental debt situation is the primary purpose of constructing an early warning index system for local governmental debt risk. Because the current study of local government debt risk in China is only at the primary stage, the risk estimation is only at the lower level of qualitative and quantitative research, which cannot intuitively derive the local government debt risk status. There- fore, if we want to understand the development direction of local government debt risk and the degree of risk in a comprehensive and systematic way, we must reasonably design the local government debt risk early warning index system and draw accurate conclusions through the analysis of empirical test results. The direct purpose of the study of local government debt risk early warning indicator system is to prevent and resolve local government debt risk, as shown in Figure 1 . Thus, in order to be able to effectively monitor the local governmental debt situation, to put forward countermeasure suggestions for preventing and resolving the potential risks of local governmental debt according to the degree of risk, and to avoid the risk of local governmental debt, the construction of the debt risk early warning index system is imperative. Maintaining the smooth operation of the economy is the ultimate purpose of constructing the local government debt risk early warning indicator system. Because of the vertical and horizontal transmission mechanism of debt risk, the expansion of debt risk will eventually have a negative impact on the fiscal balance, the financial sector's capital operation, and the prestige of the government and eventually fuse together to affect the smooth operation of the economy and the stable development of society. Therefore, for the normal development of the social economy, it is necessary to construct a risk early warning indicator system.

To ensure the rationality and scientificity of the construction of the risk early warning indicator system, it is necessary to adhere to the construction principles. The main construction principles are as follows:

(1) The principle of comprehensiveness. Early warning indicators should be comprehensive, and not just a few representative indicators, because too few indicators will easily miss some key indicators, and the constructed early warning indicator system will not guarantee effectiveness

(2) The principle of relevance. An accurate indicator system must adhere to relevance. This relevance means that it is relevant to the risk of local governmental debt and that the indicators ensure that they reflect the level of local governmental debt risk

(3) Principle of structure. The selected risk warning indicators reflect the level of local economic development, the balance of local fiscal revenues and expenditures, the risk status of the state-owned and financial sectors, the level of local government debt risk and other conditions, and the structural division of factors affecting local government debt risk

(4) The principle of realism. The early warning indicator system should not blindly draw on the experience and practices of other countries but should be consistent with the national situation of local government debt in China and can truly reflect the mechanism and characteristics of local government debt risk formation under the background of the existing system in China 

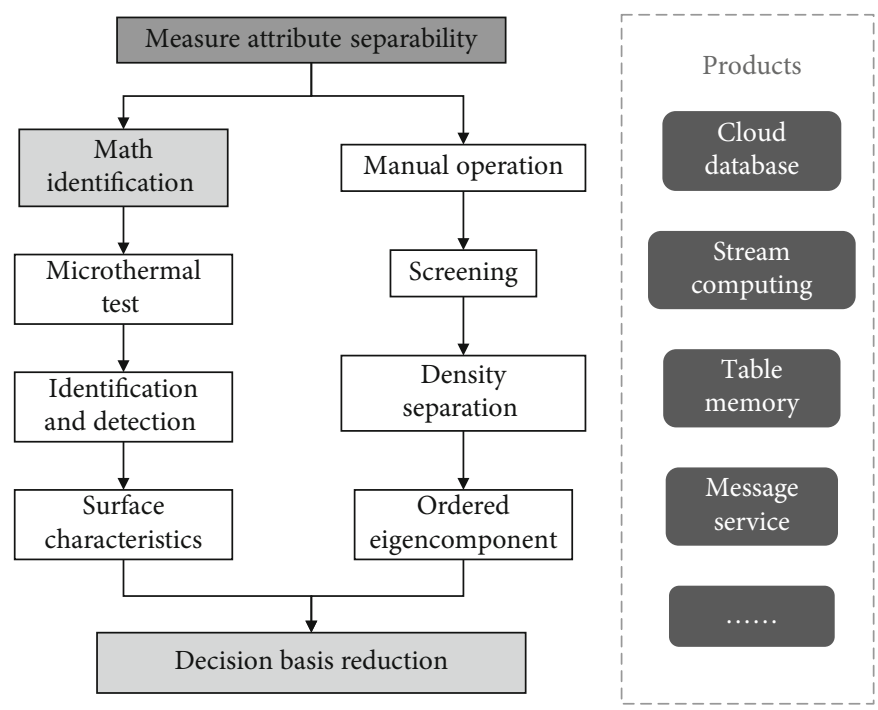

Figure 1: Early warning indicator system for local government debt risk.

The selection of local government debt risk methods is different, the samples selected will be different, the required early warning methods will be different, and the final size of the risk derived will be different. Therefore, after a comprehensive analysis of the characteristics, applicability, and operating principles of each early warning method, a reasonable early warning method is selected and conclusions with certain realistic significance are drawn. To sum up, there are two main early warning methods for local government debt risk: the single indicator method and the comprehensive indicator method.

\section{(1) Single indicator approach}

The single-indicator method refers to the selection of a few single indicators with the greatest impact from a large number of local government debt risk measurement indicators, and judging the size of local government debt risk based on the risk threshold value of each single indicator. Commonly used indicators include debt service ratio, debt dependency ratio, fiscal deficit ratio, local government debt burden ratio, debt growth rate, and other indicators. It is enough to choose one of these indicators to detect the risk of local governmental debt. This method is simple and easy to operate, only needs to obtain the relevant data of the required indicators, and combined with the international common alert value can be derived from the debt risk size. However, this method also has certain shortcomings: because each indicator has more or less influence on the degree of local government debt risk, the conclusion of just one indicator is largely one-sided, and the accuracy of the results cannot be guaranteed; the development of local government debt is a dynamic development system; only one indicator reflects the static level of risk in the current period and cannot monitor the longer-term debt risk. It is not possible to monitor the debt risk over a longer period of time.

(2) Integrated early warning method
The comprehensive early warning method is to select multiple indicators that can represent various types of risks for a systematic and comprehensive analysis of risks on the basis of a comprehensive consideration of various risk factors, so as to draw a comprehensive conclusion on the size of risks. The comprehensive evaluation method can comprehensively analyze the degree of influence of various types of indicators on government debt risk and can well compensate for the one-sidedness of single-variable models, which has become the main research method adopted by the theoretical community. At present, the three main comprehensive early warning methods commonly used for early warning of local government debt risk are the $Z$-score model, fuzzy comprehensive evaluation method, and artificial neural network evaluation method.

\section{(1) Q fraction model}

The Q-score model is a multivariate discriminant analysis model that requires specifying the values of the observed variables and determining the classification of the observed objects. First of all, many variables are censored, and then, a few variables that can reflect the discriminant information to the greatest extent are obtained by some methods, and then, a discriminant function is established for these variables obtained by screening, and the discriminant coefficients of each variable are determined, and finally, the observed values of the independent variables are brought into the discriminant function, and the discriminant values can be derived, as shown in Figure 2. The general form of the discriminant function is as follows:

$$
\begin{aligned}
Q & =q_{1} x_{1}+q_{2} x_{2}+. \cdots+q_{i} x_{i} \\
\sum_{i=1}^{n} x_{i} q_{i} & =\frac{\sqrt{\widetilde{x^{2}}}+y^{2}}{n}
\end{aligned}
$$




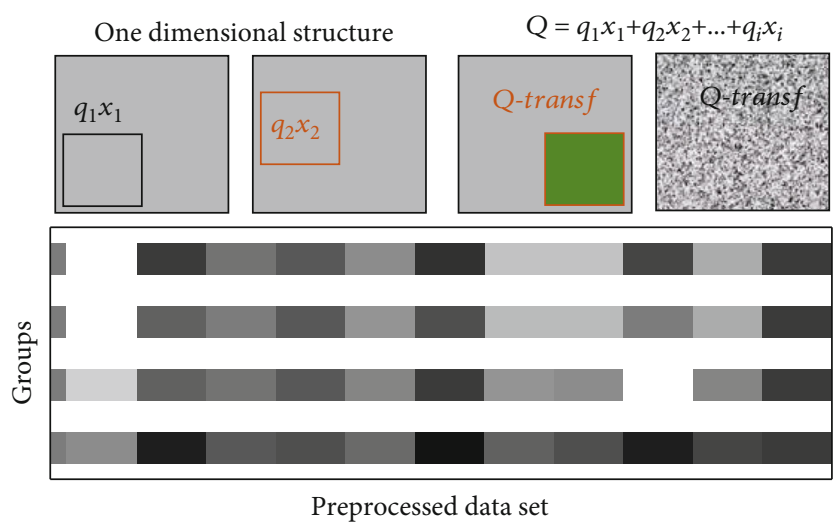

FIGURE 2: $q$-fraction model.

where $W 1, W 2$, and $W 3$ are the discriminant coefficients of each variable; $X 1, X 2$, and $X 3$ are the characteristic variables, and $Z$ is the discriminant value. The comprehensive early warning method of the $Z$-score model needs to pay attention to the determination of the discriminant coefficients of each variable, in addition to the reasonable selection of screening methods. At present, there are two commonly used methods for determining the discriminant coefficients: subjective determination method and objective determination method. The subjective determination method is an expert evaluation method, in which experts in the field determine the weight coefficients by judging the importance of the indicators in the index system, which has a greater subjective tendency; the objective determination method, such as the correlation coefficient method and factor analysis method, removes the subjective factors to a certain extent, but its reasonableness is open to question.

\section{(2) Fuzzy integrated evaluation method}

The fuzzy comprehensive evaluation method is a method based on fuzzy mathematics, which can convert qualitative evaluation into quantitative evaluation, and is a comprehensive early warning method. Whether the selection of evaluation indicators of this method is appropriate will directly affect the accuracy of the evaluation results. The specific implementation steps are as follows: first, determine the weight value of each index by using expert experience or AHP hierarchical analysis; second, establish the affiliation function and construct the evaluation matrix; third, select the suitable synthetic factors to obtain the synthetic results and interpret the synthetic results. This evaluation method was proposed in 1965 by Professor Chad (L. A. Zadeh), an American automatic control expert, to express the uncertainty of things. The advantage of the fuzzy synthesis evaluation method is that it has clearer evaluation results and stronger systematization, which is advantageous for dealing with fuzzy and difficult to quantify problem bureau. Since it was proposed, it has been widely used in many fields such as robotics, process control, subway locomotives, fault diagnosis, medical diagnosis, sound recognition, image processing, bidding, and quotation. A major difficulty of the fuzzy comprehensive evaluation method is about the determina- tion of evaluation indicators, and the current common method of determining the weights is the expert evaluation method or using the same weights for each indicator, which is more subjective and affects the accuracy of the early warning results.

(3) Early warning methods based on artificial neural networks

Artificial neural network early warning methods originated in the field of artificial intelligence, which is actually an operational model formed by a large number of interconnected neurons (or nodes). Each neuron can represent a specific class of output functions, which can also be called excitation functions. Every arbitrary connection between two neurons represents a weight value of the signal passing through that connection. The output of the artificial neural network differs depending on the choice of connection, weight value, and type of output function of the network. The early warning method does not require a strict distribution of data and has the ability to handle information omission. Comprehensive analysis of the above, using the integrated early warning method is more scientific and reasonable than the results derived from a single early warning method and can better ensure the effectiveness of the early warning results, and the government debt risk early warning model currently established in China mainly uses the linear relationship between indicators, while in fact, the early warning system is a multifaceted, nonlinear, and complex determination system. Among the comprehensive early warning methods, the artificial neural network-based early warning method has the characteristics of nonlinearity, fault tolerance, and generalization ability compared with the other two early warning methods, which not only excludes the tendency of subjectivity but also can eliminate the factor of colinearity, and thus has more advantages.

3.2. Public Crisis Management Exception Warning. A public crisis is a type of crisis that is more impactful and damaging. Generally speaking, no matter what type of crisis, events that involve the interests of the majority of the public, potentially or in fact generate undesirable social, economic, or political problems, and require government involvement or governance to contain the expansion of the crisis are public crises. From a philosophical perspective, crises and public emergencies are related in a general and specific way. According to the manifestations of sudden public events, crises can be divided into natural disasters, catastrophic accidents, public health events with a sudden impact and sudden social security responsibility events, and finally, economic and political crises. As for the specific definition of a public crisis, a public crisis is a situation in which people's common and important life goals are interrupted in the face of a public emergency, as shown in Figure 3. In short, that is, through a certain method is the original gray information into white information; the advantage is that there is no requirement for the number of samples and it does not need to obey any specific distribution, especially in the case of a small amount of data or when the calculation conditions are not met, it can better reflect its advantages. The term "impeded condition" is 


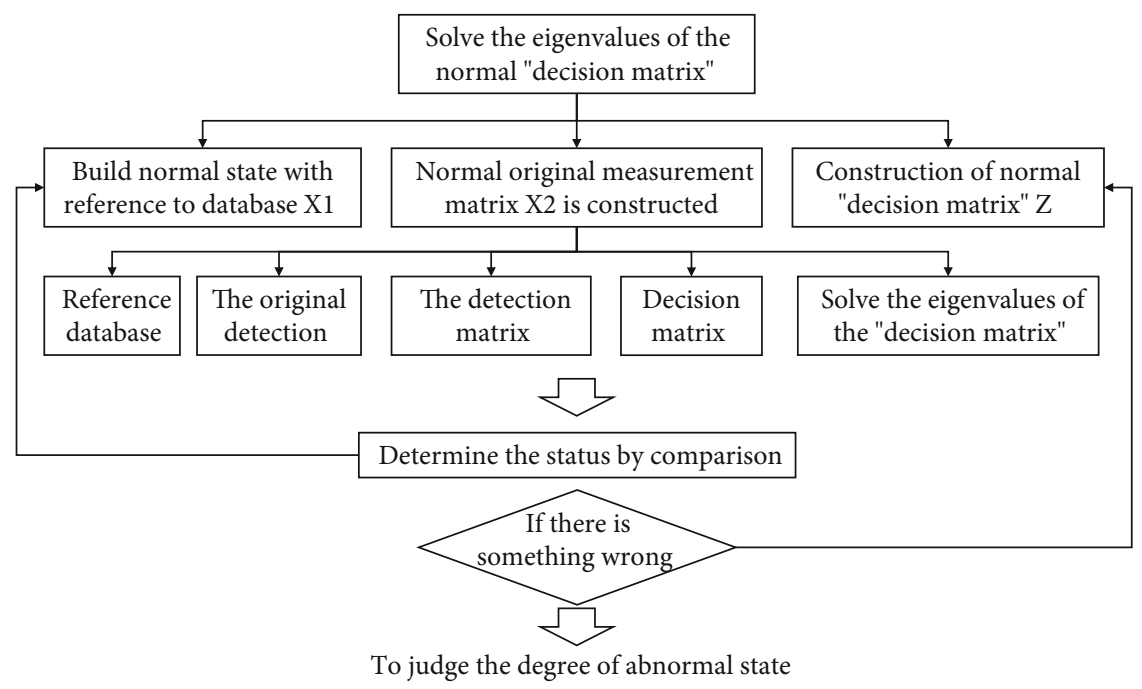

FIGURE 3: Early warning of public crises.

used here to refer to the inability of individuals or small groups of people to change or improve the situation in the face of a public emergency; it further emphasizes the inevitability and public nature of public crises. Robert McNamara, a former U.S. Secretary of Defense and President of the World Bank, suggested that "the strategy of the future may cease to exist and will be replaced by public crisis management." This statement fully reflects the importance of public crisis management, which has changed from a single emergency management to a multifaceted collaborative social crisis management, and further illustrates the importance of public crisis early warning. Once a public crisis breaks out, the normal way of functioning of the members of the society in the region to which it belongs will be greatly affected or even destroyed, and numerous social relationships will be out of balance, which in turn will affect a wider scope or even a society-wide change in the environment. Compared to ordinary crises, public crises are extremely socially harmful, uncontrollable, significant, and widespread."

A public crisis is a state of emergency in which events occur suddenly under uncontrollable circumstances, causing threats and damage to the normal operation of society and the order of survival of the people, life, and property. For example, the Sanlu tainted milk powder incident, the Wenchuan earthquake, the Shanxi mining disaster, and other crises that cause explicit or invisible harm to human life can be classified as public crises. Although public crises are unpredictable and difficult to prevent and control, there are still special laws that can be explored and used. The importance and necessity of public crisis management have emerged by exercising human initiative; grasping these laws, coupled with feasible and operable measures and coordination, public crises can be effectively controlled. Different scholars have given different understandings of public crisis management. According to Steven Fink, an American crisis management expert, public crisis management is "the art of systematically removing risk and uncertainty from a crisis at a turning point in an organization's future, so that the organization is more in control of its own future."

It can be seen that the earliest crisis management in business management provides a good way to respond to the "principle of exception" for leaders, and it can also be seen as an art of leadership. As the application of public crisis has expanded, public crisis theory has emerged in more areas. Xia Shuzhang, a political scientist in China, pointed out that "public crisis management is a dynamic process, including pre-crisis early warning management, crisis emergency treatment and post-crisis aftercare, which is an all-round management behavior. The tasks of crisis management include four aspects: dealing with accidents, controlling the situation, coordinating relations, and reshaping the image." These scholars' views provide a template and basic starting point for the study of public crisis management in China, as well as a model for our government to follow to enhance its ability to respond to crises.

Crisis management is a part of government management, and public crisis management is a part of crisis management, so public crisis management becomes a duty of the government and an important way for the government to enhance its image and trust. Looking at the views of many scholars, the author defines public crisis management as follows: public crisis management refers to a new dynamic management process in which public organizations make scientific decisions and deal with and summarize public crisis cases through reasonable monitoring of crises, with the aim of eliminating or reducing the threats and losses caused by crises, thus making crisis handling scientific and systematic. Public crisis management, sometimes referred to as government crisis management, is the management activities carried out by the government in response to public crisis events and is a solution to the sudden and devastating problems in the government's external interactions and internal management. Public crisis management initially arose and was applied in business management. Along with the deepening of government administrative 
reform, the government constantly has to introduce the advanced management concepts of enterprises in order to establish a service-oriented government, and public crisis management has developed and grown to become an important function of the government. Coupled with the increasing socialization and globalization of crisis events, the importance and urgency of public crisis management research have attracted the common attention of all countries and active concerted cooperation.

\subsection{Risk Early Warning Models and Evaluation Indicator} Systems. Debt is an important channel and means to raise construction funds, but it is a "double-edged sword"; if used properly, it can promote economic and social development; otherwise, it will form a heavy debt burden and increase the risk of government debt. 2010 onwards, due to the implementation of macrocontrol policies by the central government, the economic growth rate has slowed down and the uncertainty of the real estate market, resulting in the local government debt problem, especially the township government debt uncertainty in the real estate market, resulting in local government debt, especially township government debt, has become increasingly prominent. The long-term accumulation of township government debts will have serious implications for the future financial operations, economic development, and social stability of township-level governments once debt risks and crises are formed. In order to detect and effectively solve the debt and financial risks of the township-level government in a timely manner, to adapt the scale of debt to the social and economic development, and to give full play to the role of debt in promoting economic development, it is necessary to establish a set of guiding financial risk evaluation models for township governments based on the debt risk index system, to guide township governments to accurately grasp and scientifically analyze the debt situation, so as to keep the scale of debt control within a bearable range and maintain a good quality of financial and economic operation.

The evaluation of the financial and debt risks of township governments is a comprehensive, complex, multifactor, multiobjective, and integrated problem. Since multiple factors or indicators will play a role in the evaluation process, the existing evaluation becomes a comprehensive judgment under the joint influence of multiple factors, and the problem becomes a problem with universal significance encountered in the multifactor decision-making process, where evaluation is for decision-making, and decision-making cannot be separated from evaluation. Comprehensive evaluation is the premise of scientific decision-making and is an important basic work in scientific decision-making. Comprehensive evaluation, in short, is to adopt a certain method or means to all evaluation objects, based on the existing or given conditions, to assign values to each object, and to rank according to the assigned values. The problem of evaluating the financial risk of the township government involved in this paper can actually be summarized as a comprehensive multi-indicator evaluation problem, through a certain comprehensive evaluation mathematical function, combining multiple indicators into a comprehensive indicator; there are many methods of combination; the key lies in how to choose a more appropriate method according to the decision-making needs and the characteristics of the evaluated system. In the middle of the last century, fuzzy mathematics was widely used in comprehensive evaluation, and with it came the new method of fuzzy comprehensive evaluation of qualitative indicators. The 1970s-1980s was the era of modern scientific evaluation, during which a variety of widely used evaluation methods, such as hierarchical analysis and data envelopment analysis, were produced. 1980s-1990s was the era of modern scientific comprehensive evaluation, in which various fruitful researches on evaluation theories, methods, and applications were carried out, such as the application of artificial neural network technology and grey system theory applied to comprehensive evaluation.

In traditional cybernetics, a black system characterizes information that is unknown, a white system characterizes information that is clear, and a gray system characterizes an intermediate system between the two. This type of system is usually information-poor, and traditional evaluation methods are not effective. The object of study is an information-poor uncertain system, and the processing and development of known information is usually used to achieve an accurate description of the system. Gray system theory mainly adopts the new method of correlation degree analysis, after determining the data series, calculates the correlation degree according to the corresponding formula, and reflects the closeness of different evaluation targets to the standard, or its degree of merit, and the evaluation target with the largest correlation degree is determined as the best. The above situation can be attributed to the incompleteness of information systems, that is, "grayscale." This method starts from the incompleteness of information and the complexity of the system and analyzes the data at a certain level through mathematical methods to understand the trends and the related checks and balances. Relevance analysis is the main element of the theory, and it is a simple, reliable, and adaptable method to characterize the strengths and weaknesses of different evaluation objects by determining the degree of relevance.

\section{BP-Based Artificial Neural Network for Risk Management and Analysis}

The computational process of storing the internal laws of information in the weight matrix during the training process of the network is called learning. The basic computational element of an artificial neural network is the neuron, which is a characterized simple description of the neuronal structure of the human brain, with the main component being a nonlinear element consisting of multiple sets of inputs and a single set of outputs, the structure of which is shown in Figure 4.

In the above figure, $u_{i}$ characterizes the internal state of a neuron $i, x j$ is the input layer signal, $\theta i$ is the threshold, $w i j$ is the weight of each input layer connected to the neuron, and $\mathrm{Si}$ is the control signal of some external input. The neuron model shown in the figure is often described by a firstorder differential equation, expressed by simulating the 


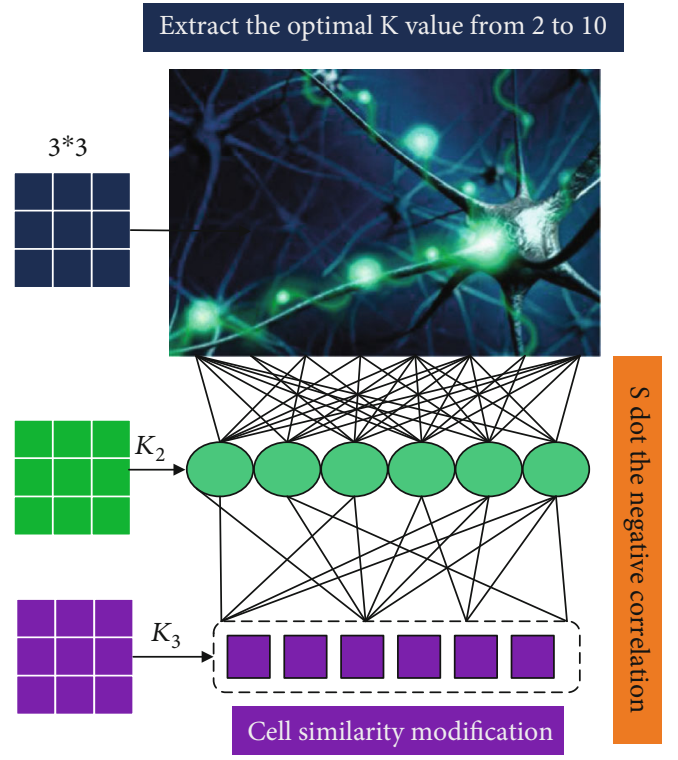

Figure 4: Neuron model.

change in synaptic potential of a biological neural network over time as

$$
\begin{aligned}
t \sum_{i=1}^{n} x_{i} q_{i} & =\frac{\partial u_{i}}{\partial t}+u_{i}(x), \\
x_{i}(t) & =f\left[u_{i}(t)\right] .
\end{aligned}
$$

The activation function of the neuron output is denoted by $f$. The following expression is often used to represent the nonlinear characteristics of artificial neural networks:

(1) Jumping type, i.e., step function

$$
\begin{aligned}
& x_{i}(t)=\left\{\begin{array}{l}
0, t>1, \\
1, t \leq 1,
\end{array}\right. \\
& u(t)=\left\{\begin{array}{l}
0, t>1, \\
1, t \leq 1 .
\end{array}\right.
\end{aligned}
$$

(2) S-type, i.e., Sigmoid function

$$
f(u)=\frac{1}{1+e^{(-u / c)^{2}}},
$$

where $c$ is a constant. This function can reflect the saturation property of neuron, and because its function is continuously derivable, adjusting the parameters of the curve can obtain a function similar to the leap-function. Therefore, this function is widely used in the output characteristics of a variety of neurons. The artificial neural network model technology is derived from people's research on the processing and judgment mode of information by the neural network sys- tem of human brain. Through the deep understanding and reasonable simplification of the working principle of human brain, the artificial neural network model similar to the thinking, learning, and training mode of human brain is designed and improved, and the output of the final calculation result is realized by simulating the neuron structure, signal transmission, and processing of human brain, as shown in Figure 5. In general, neurons are the basic components of artificial neural networks, and a large number of neurons constitute a nonlinear dynamic system through combination, whose main characteristics are high parallel computing capability, strong information processing capability, and dynamic feedback capability.

The learning process of artificial neural networks, which can also be referred to as the training process, focuses on optimizing the different parameters of the network (including weights and thresholds) by the action of the stimuli to which the network is subjected, prompting the neural network to respond to external stimuli and the environment. Learning based on sample information and environmental stimuli and improving its performance during the learning process are precisely the most valuable qualities of the method. Depending on the different ways in which the learning process is organized, learning approaches can be divided into two categories, namely, supervised and unsupervised learning, as shown in Figure 6. For supervised learning, a certain number of training samples, called training pairs or training sets, need to be prepared for the learning and training of artificial neural networks. During the learning process, not only the input patterns need to be given but also the corresponding target patterns at the output, called mentor signals, i.e., desired outputs. During the learning training, the input patterns in the training set are adopted one by one and the actual output patterns are computed according to the model and their errors are obtained after comparing with the mentor signal. The weight matrix is then adjusted according to the error and the model algorithm, which aims to reduce the error. By continuously using different training sets and iterating this computational model repeatedly, the computational results are obtained under the condition that the error is lower than the desired error by adjusting the weights and thresholds, etc.

The computational process of storing the internal laws of information in the weight matrix during the training process of the network is called learning. This process requires not only storing the corresponding information but also summarizing the internal laws of the information. The process of presenting the learned internal laws in the output layer of a trained artificial neural network under the action of an input pattern can be called association (or memory). The aforementioned patterns of stored intrinsic laws can be divided into two categories: spatial and temporal graphs. Association is one of the main features of the human brain and can also be divided into self-association and association with others. An association that associates all the features of a thing from a part of the thing is called self-association, while an association from one thing to another is an association of others, as shown in Figure 7. The learning of artificial neural network is mainly based on some training rules 


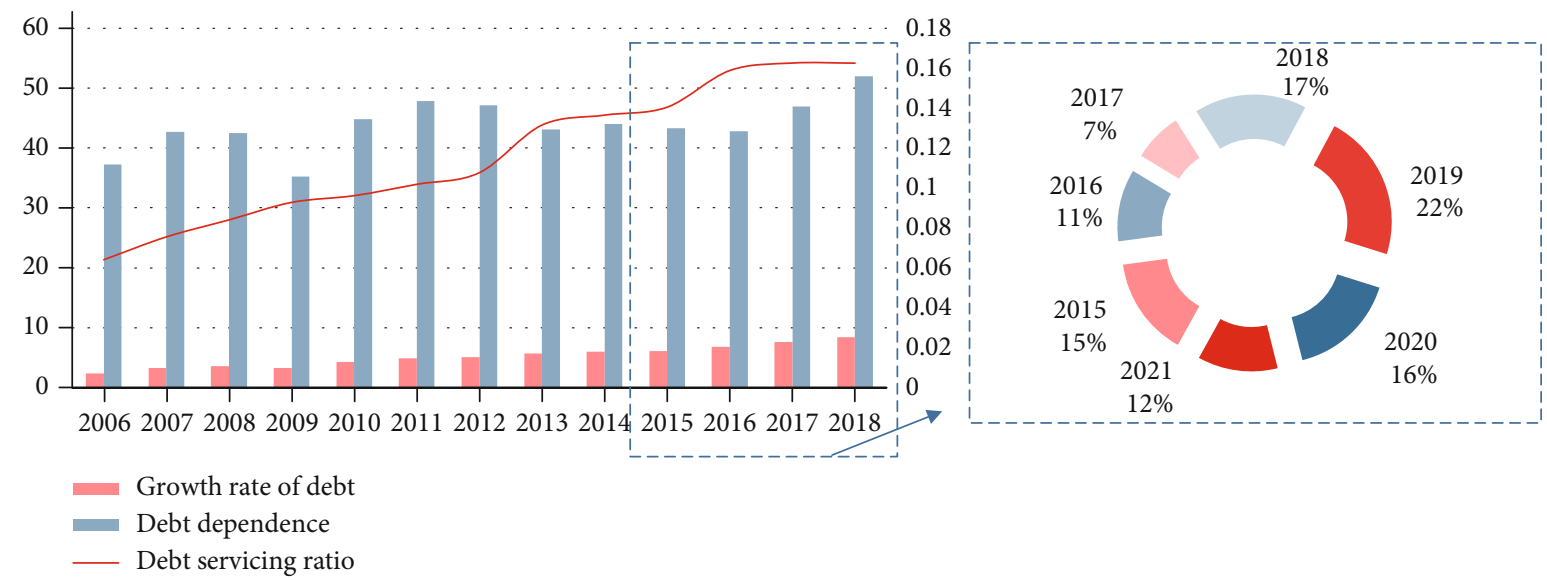

FIgURE 5: Output of calculation results.
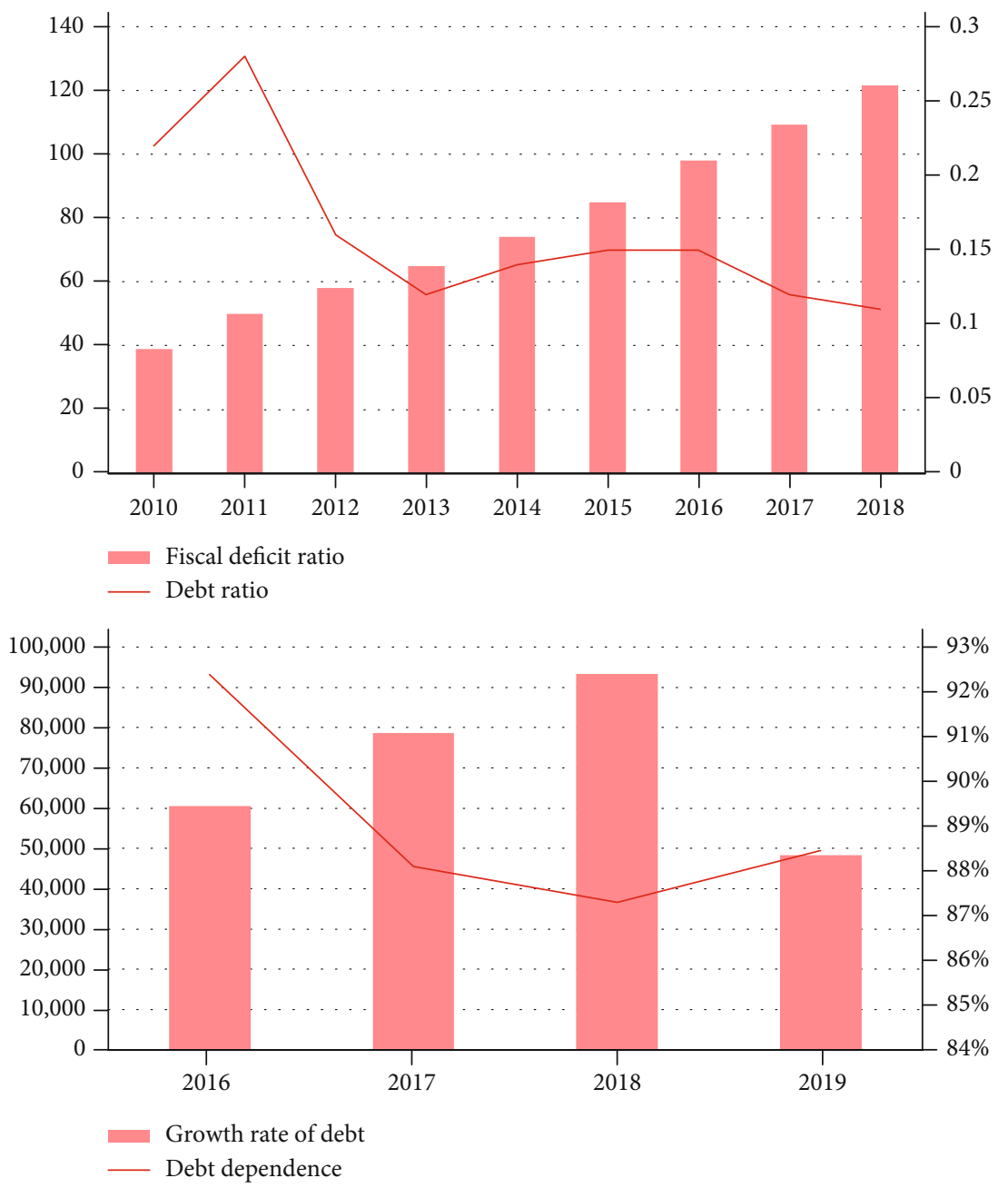

FIgURE 6: Training output results.

determined by the model, and its memory function is realized by dynamically modifying the values of connection weights between units and storing the specific pattern distribution of the learned content in the structure of the computational model. Repeating the above process, new patterns of other contents can also be learned. There are dozens of models of artificial neural development so far, but in general, they can still be divided into three categories: forward networks, feedback networks, and self-organizing networks, and the BP artificial neural network with error 


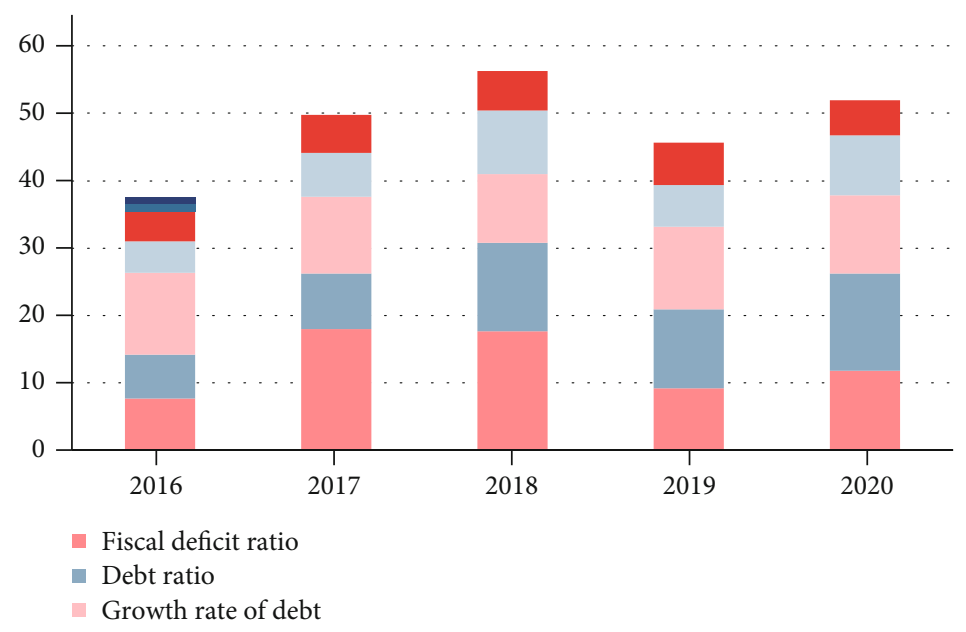

Figure 7: Output of triple association results.

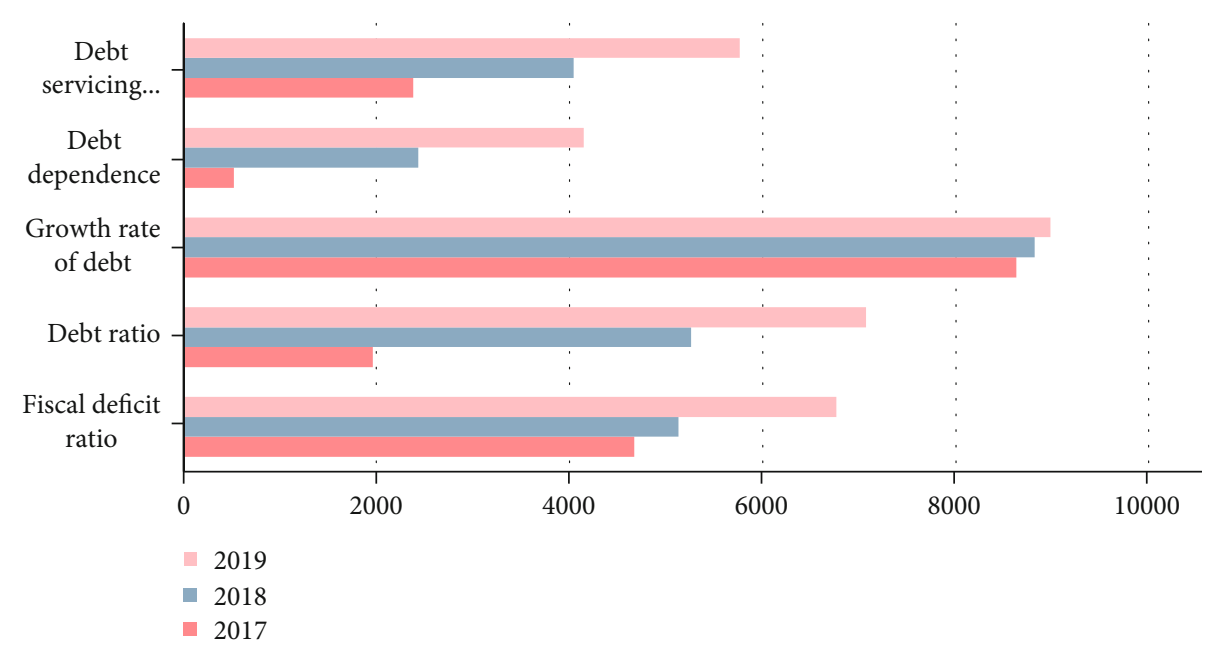

FIGURE 8: Risk interval values of financial risk evaluation indicators for township governments.

back propagation algorithm proposed in the mid-1980s is still one of the most widely used models so far and is the core part of the forward network, representing the most perfect and essence of the artificial neural network model.

The above indicators take GDP as the main base variable, the indicators contained in the two subsystems of debt characteristics and debt servicing capacity as the main indicators, and the socioeconomic subsystem and the indicators contained therein as auxiliary indicators, and the scale risk, change risk, and debt servicing risk of the township government debt are considered comprehensively, with the aim of comprehensively analyzing the debt pressure faced by the township government and accurately reflecting the financial and debt risks of the township government risk, as shown in Figure 8. In order to determine the weight values of the above seven risk evaluation indicators, this study designed a questionnaire and distributed 90 copies to experts or practitioners in related fields (such as staff of relevant government departments, experts from universities and research institutions, and master and doctoral students from universities) in order to obtain comparative findings on the relative importance of different indicators. The actual number of valid questionnaires collected and collated was 75 .

\section{Conclusion}

Focusing on the financial risks of township governments caused by debt risks, this paper starts from the definition of the concepts of financial risks and debt risks of township governments, summarizes the characteristics of debt and financial risks of township governments, and analyzes the main causes of the above risks from the aspects of economic system, financial system, and debt management. Based on the above analysis and summary, this paper draws on the idea of traditional comprehensive evaluation and applies it to the construction of comprehensive evaluation indexes for the early warning system of township government financial risk. In calculating and evaluating the debt risk of township governments, the debt risk is decomposed into several interrelated and independent individual risk indicators, mainly including fiscal deficit rate, debt burden rate, debt ratio, debt dependency, debt growth rate, debt service rate, 
and debt overdue rate. And according to the importance degree of the above risk indicators in the overall debt risk, the weight of them in describing the risk of township government debt was determined by using hierarchical analysis. Finally, the overall indicators for the comprehensive evaluation of the fiscal risk early warning model of the township government are then obtained through the comprehensive weighted average calculation method. This paper argues that what is lacking in the current fiscal risk early warning model is precisely the area where BP artificial neural networks are good at, such as pattern processing, function approximation, and optimization processing. The early warning indicators correspond to the input layer data of BP artificial neural network, and the risk intervals correspond to the results obtained after the risks are classified, and it can be seen through the study that local government debt risk early warning is suitable for the use of neural networks. In this paper, the model of township government financial risk early warning is constructed by using the BP neural network method, and a visual user interface based on Matlab language is written, which is convenient for model setting and sample data input, and the human-computer communication is smoother.

In the future, individual local governments have established a risk early warning mechanism, and the debt risk information disclosure system is not sound, so it is inevitable that there is a lack of clear judgement on the size of local government debt risk.

\section{Data Availability}

The data used to support the findings of this study are available from the corresponding author upon request.

\section{Conflicts of Interest}

The author declares no known competing financial interests or personal relationships that could have appeared to influence the work reported in this paper.

\section{References}

[1] A. Allahdadi, D. Pernes, J. S. Cardoso, R. Morla, and H. Markov, "Hidden Markov models on a self-organizing map for anomaly detection in 802.11 wireless networks," Neural Computing and Applications, vol. 33, no. 14, pp. 8777-8794, 2021.

[2] Y. Chang, X. Yuan, B. Li, D. Niyato, and N. Al-Dhahir, "A joint unsupervised learning and genetic algorithm approach for topology control in energy-efficient ultra -dense wireless sensor networks," IEEE Communications Letters, vol. 22, no. 11, pp. 2370-2373, 2018.

[3] M. Chen, K. Liu, J. Ma et al., "Moloc: unsupervised fingerprint roaming for device-free indoor localization in a mobile ship environment," ieee internet of things journal, vol. 7, no. 12, pp. 11851-11862, 2020.

[4] W. Cui, K. Shen, and W. Yu, "Spatial deep learning for wireless scheduling," ieee journal on selected areas in communications, vol. 37, no. 6, pp. 1248-1261, 2019.
[5] M. Eisen and A. Ribeiro, "Optimal wireless resource allocation with random edge graph neural networks," ieee transactions on signal processing, vol. 68, pp. 2977-2991, 2020.

[6] J. Gao, C. Zhong, X. Chen, H. Lin, and Z. Zhang, "Unsupervised learning for passive beamforming," ieee communications letters, vol. 24, no. 5, pp. 1052-1056, 2020.

[7] J. He, K. Lippmann, N. Shakoor, C. Ferrigno, and M. A. Wimmer, "Unsupervised gait retraining using a wireless pressure-detecting shoe insole," gait \& posture, vol. 70, pp. 408-413, 2019.

[8] M. A. Jamshed, F. Heliot, and T. W. C. Brown, "Unsupervised learning based emission-aware uplink resource allocation scheme for non-orthogonal multiple access systems," ieee transactions on vehicular technology, vol. 70, no. 8, pp. 76817691, 2021.

[9] D. Karagiannis and A. Argyriou, "Jamming attack detection in a pair of Rf communicating vehicles using unsupervised machine learning," vehicular communications, vol. 13, pp. 56-63, 2018.

[10] Y. Li, X. Hu, Y. Zhuang, Z. Gao, P. Zhang, and N. El-Sheimy, "Deep reinforcement learning (Drl): another perspective for unsupervised wireless localization," ieee internet of things journal, vol. 7, no. 7, pp. 6279-6287, 2020.

[11] D. Liu, C. Sun, C. Yang, and L. Hanzo, "Optimizing wireless systems using unsupervised and reinforced-unsupervised deep learning," ieee network, vol. 34, no. 4, pp. 270-277, 2020.

[12] S. Liu, Z. Wei, B. Li, and C. Zhao, "Unsupervised clustering-based non-coherent detection for molecular communications," ieee communications letters, vol. 24, no. 8, pp. 1687-1690, 2020.

[13] N. Nguyen, K. W. Choi, L. Song, and Z. Han, "Roommates: an unsupervised indoor peer discovery approach for Lte D2d communications," ieee transactions on vehicular technology, vol. 67, no. 6, pp. 5069-5083, 2018.

[14] S. Rajendran, W. Meert, V. Lenders, and S. Pollin, "Unsupervised wireless spectrum anomaly detection with interpretable features," ieee transactions on cognitive communications and networking, vol. 5, no. 3, pp. 637-647, 2019.

[15] L. H. A. Reis, L. C. S. Magalhães, D. S. V. de Medeiros, and D. M. F. Mattos, "An unsupervised approach to infer quality of service for large-scale wireless networking," Journal of Network and Systems Management, vol. 28, no. 4, pp. 1228-1247, 2020.

[16] D. Sikeridis, B. P. Rimal, I. Papapanagiotou, and M. Devetsikiotis, "Unsupervised crowd-assisted learning enabling location-aware facilities," ieee internet of things journal, vol. 5, no. 6, pp. 4699-4713, 2018.

[17] H. Song, M. Zhang, J. Gao, and C. Zhong, "Unsupervised learning-based joint active and passive beamforming design for reconfigurable intelligent surfaces aided wireless networks," ieee communications letters, vol. 25 , no. 3, pp. $892-$ 896, 2021.

[18] J. Wang, C. Jiang, H. Zhang, Y. Ren, K.-C. Chen, and L. Hanzo, "Thirty years of machine learning: the road to pareto-optimal wireless networks," ieee communications surveys and tutorials, vol. 22, no. 3, pp. 1472-1514, 2020.

[19] S. Xue, Y. Ma, N. Yi, and R. Tafazolli, "Unsupervised deep learning for $\mathrm{Mu}$-Simo joint transmitter and noncoherent receiver design," ieee wireless communications letters, vol. 8, no. 1, pp. 177-180, 2019. 
[20] M. U. Younus, M. K. Khan, M. R. Anjum, S. Afridi, Z. A. Arain, and A. A. Jamali, "Optimizing the lifetime of software defined wireless sensor network via reinforcement learning," ieee access, vol. 9, pp. 259-272, 2021.

[21] L. Zhang, Y. Hua, S. L. Cotton, S. K. Yoo, C. R. C. M. da Silva, and W. G. Scanlon, "An Rss-based classification of user equipment usage in indoor millimeter wave wireless networks using machine learning," ieee access, vol. 8, no. 1, pp. 14928-14943, 2020 . 